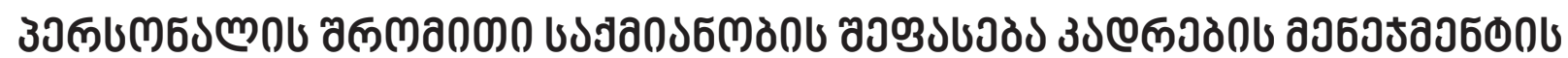

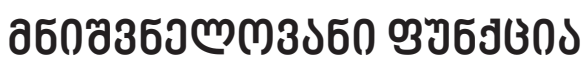

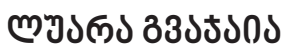

https://doi.org/10.35945/gb.2017.04.021

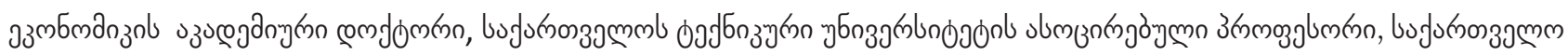

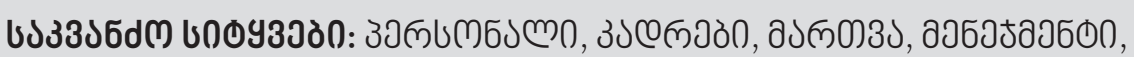

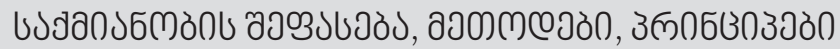

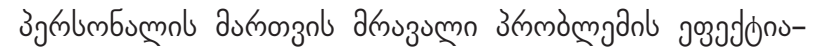

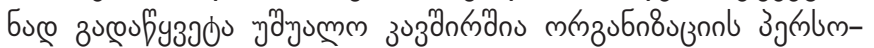

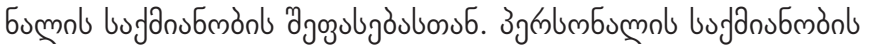

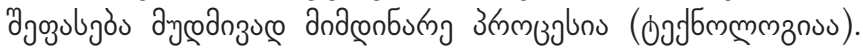

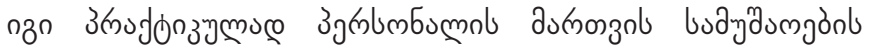

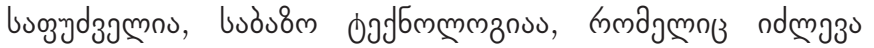

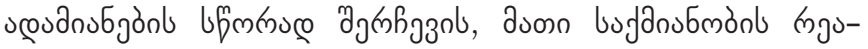

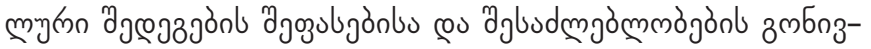

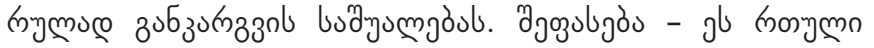

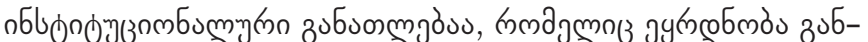

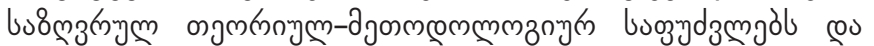

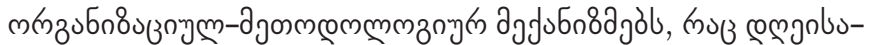

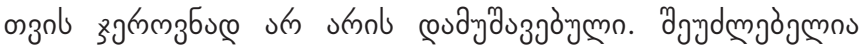

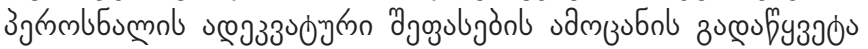

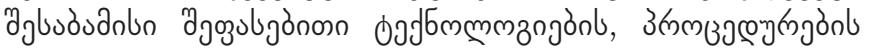

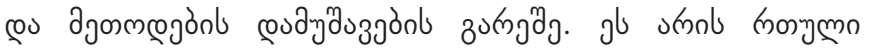

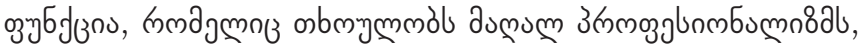

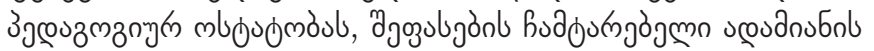

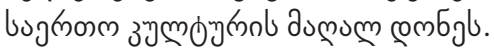

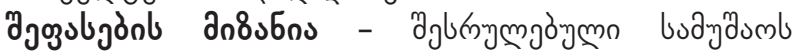

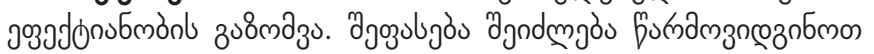

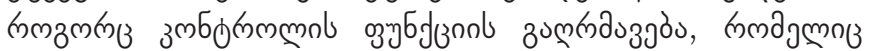

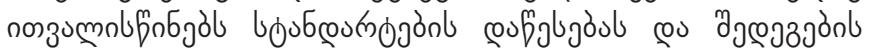

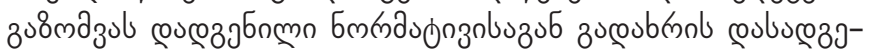

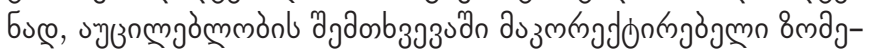
onls zodutngoul.

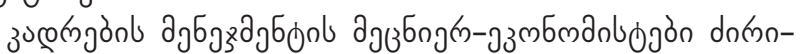

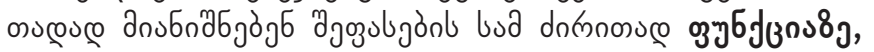
jugbns:

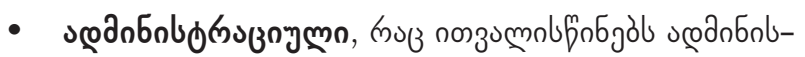

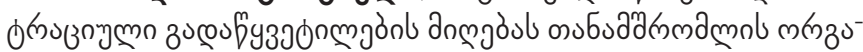

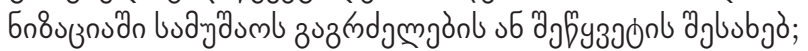

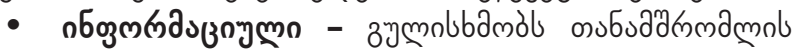

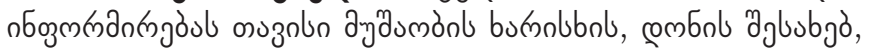

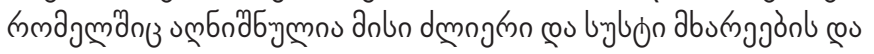

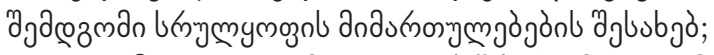

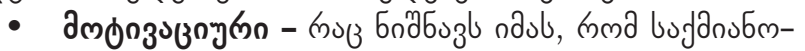

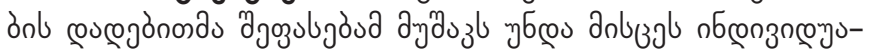

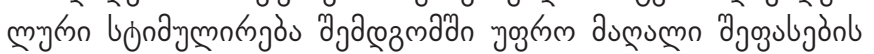
anluspipozue.

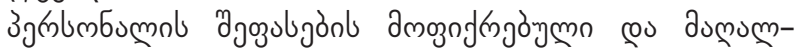

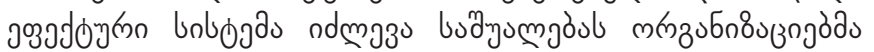

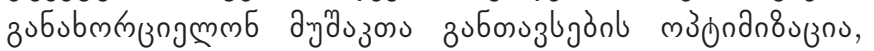

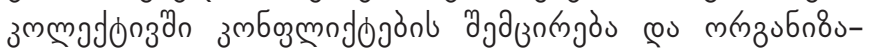

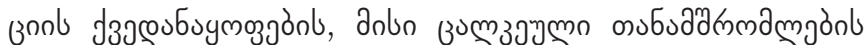
bufanobmònb sbumoßn.

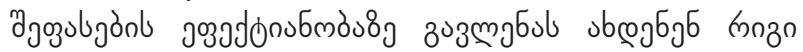

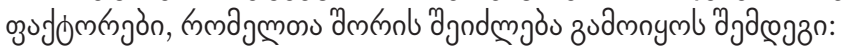

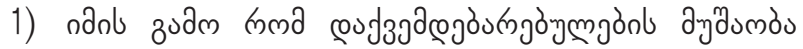

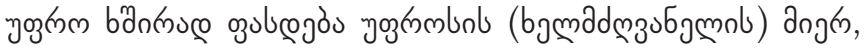
sandma

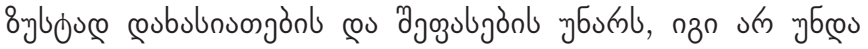

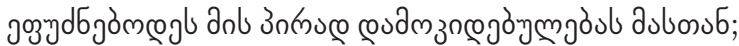

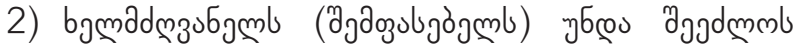

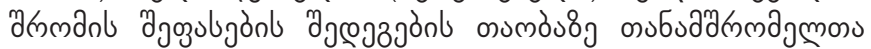

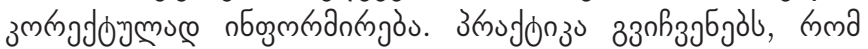

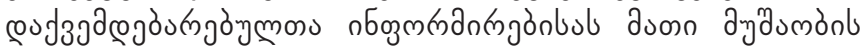

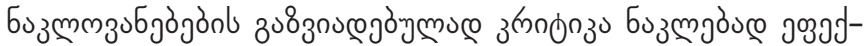

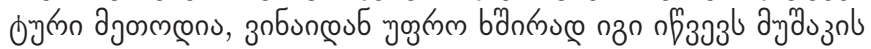

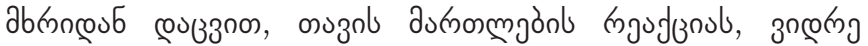

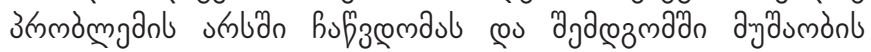

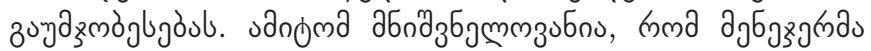

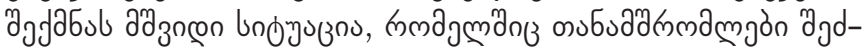

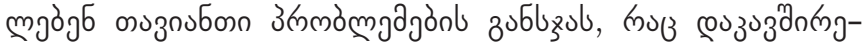

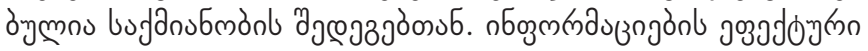

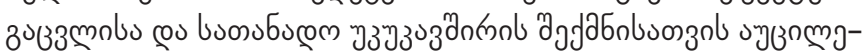

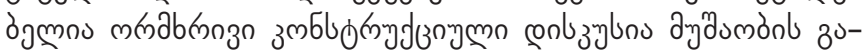

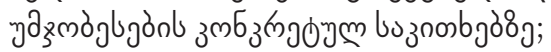

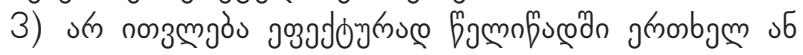

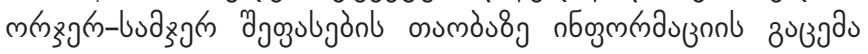

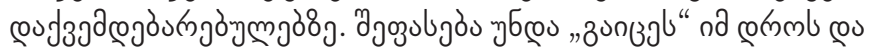

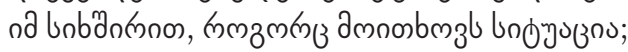

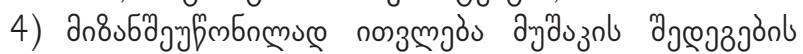

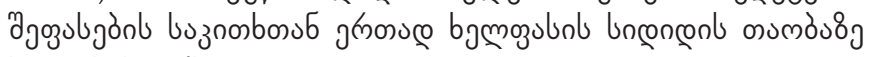
laznorbol zubbngmza.

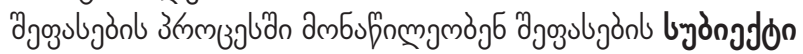
एo mónjon.

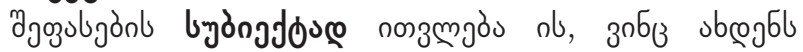

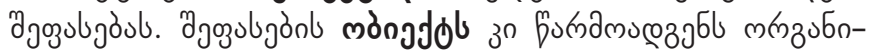

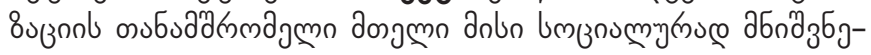

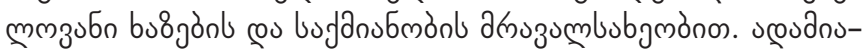

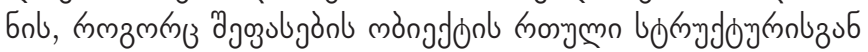

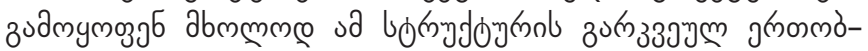
mnmbul: змб з

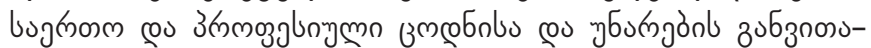

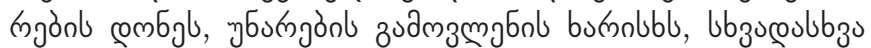

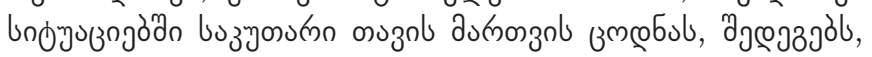

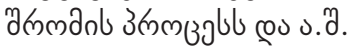




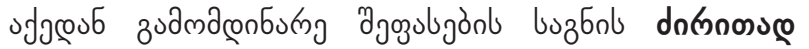

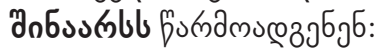

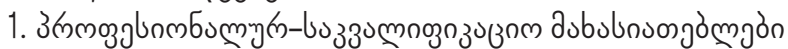

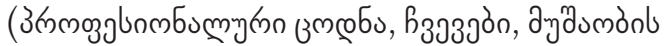

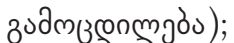

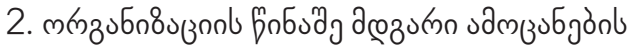

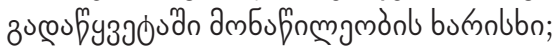

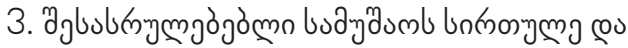

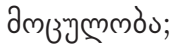

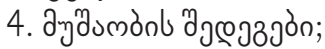

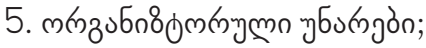

6. змm

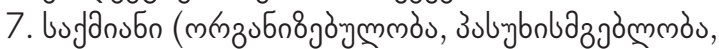

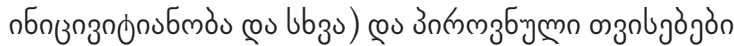

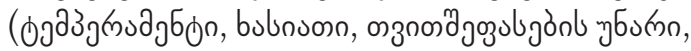

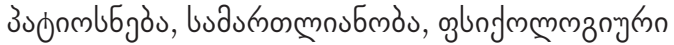

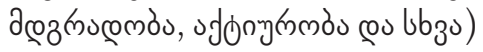

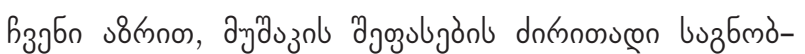

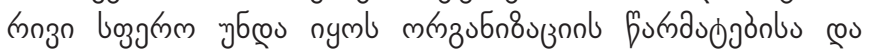

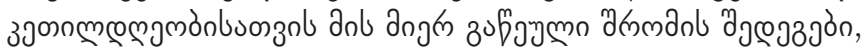

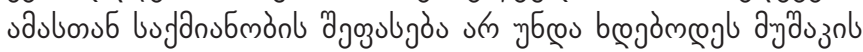

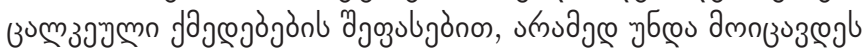

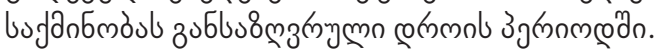

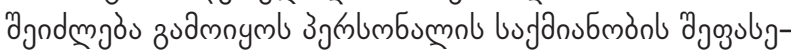
on zublus

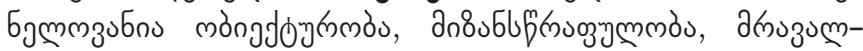

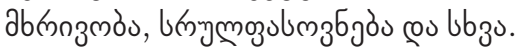

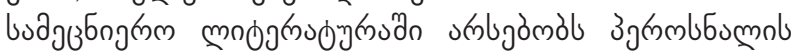

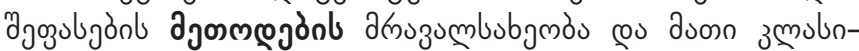

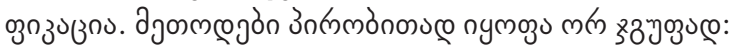

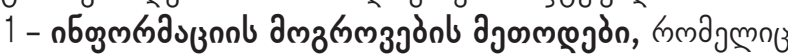

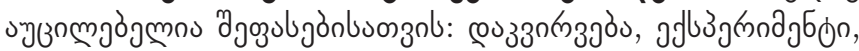

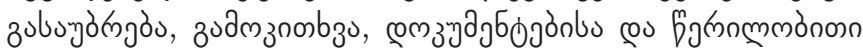

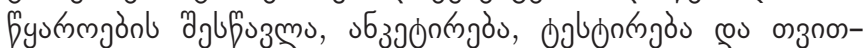
gyoguljós.

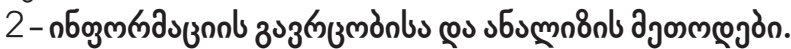

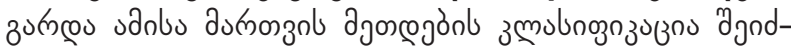

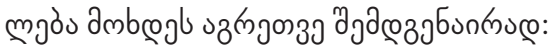

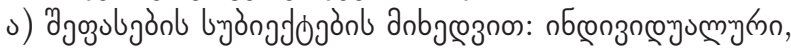

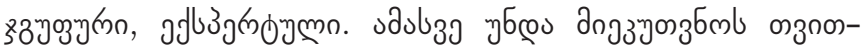

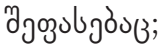

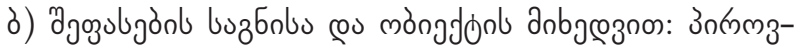

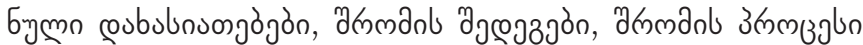
cos. o. .

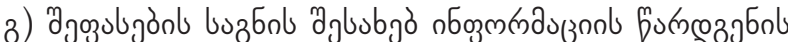

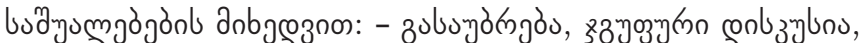

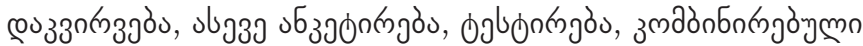

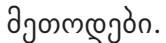

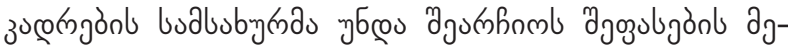

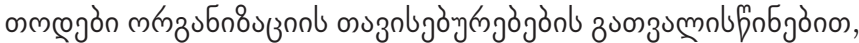

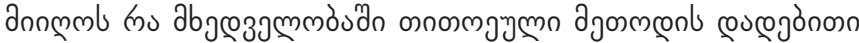

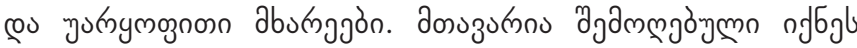

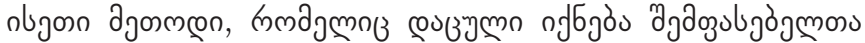

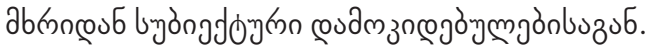

зuलng

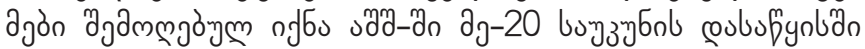

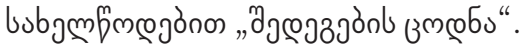

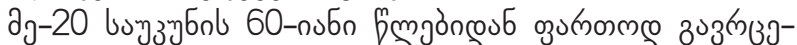

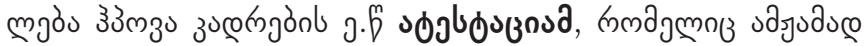

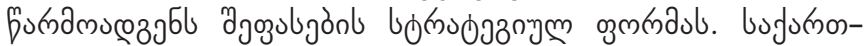

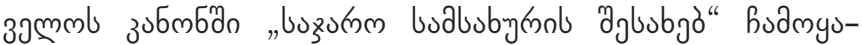

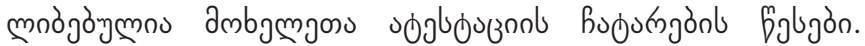

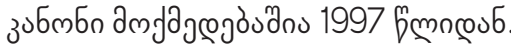

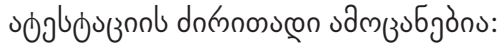

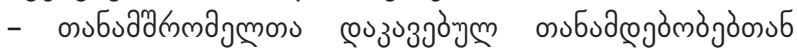

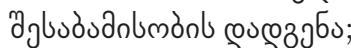

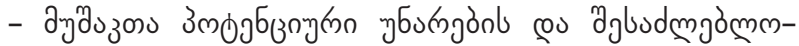

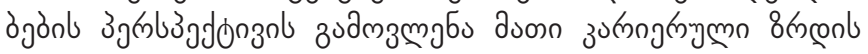
aumonaluonzol;

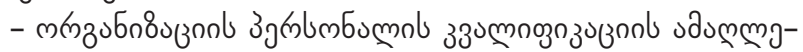

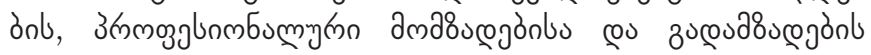

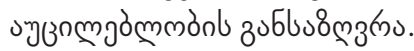

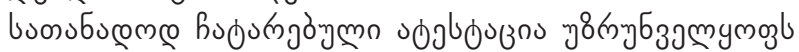

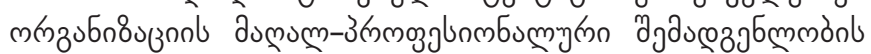

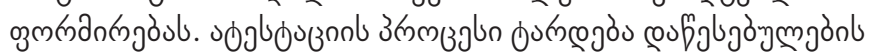

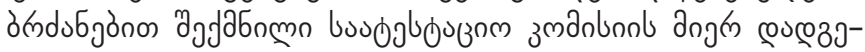

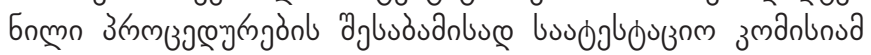

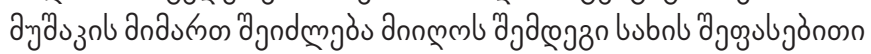
зucoufysjonngàjón:

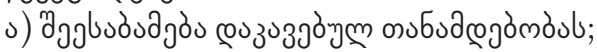

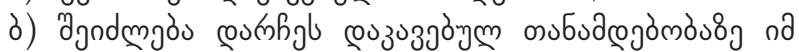

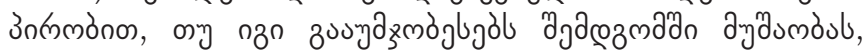

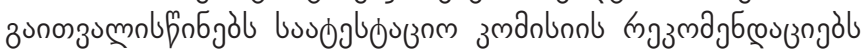

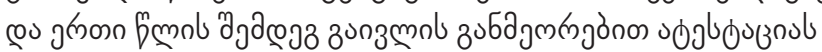

3) उल चू

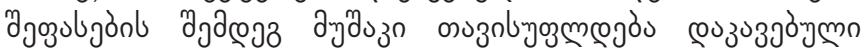

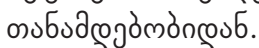

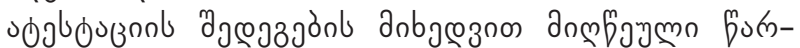

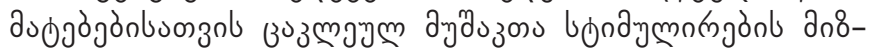

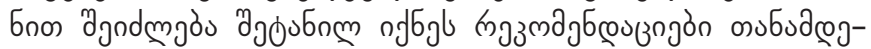

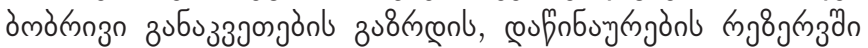

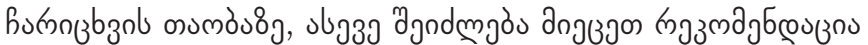

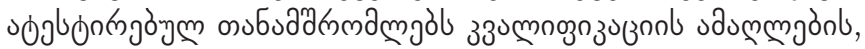

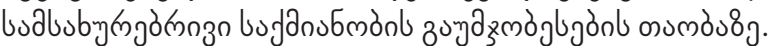

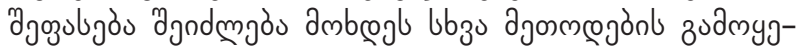

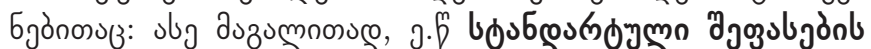

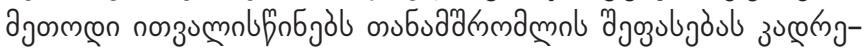

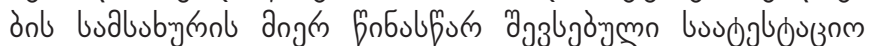

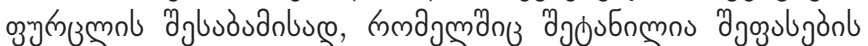

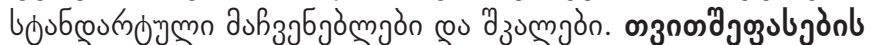

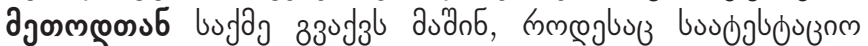

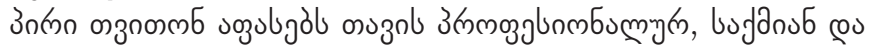

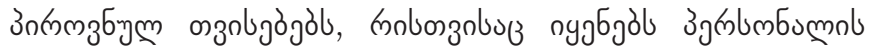

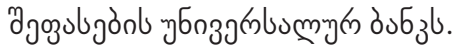

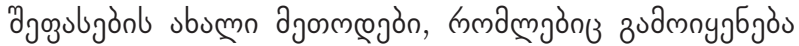




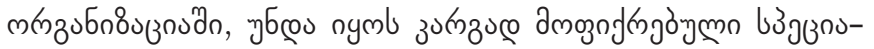

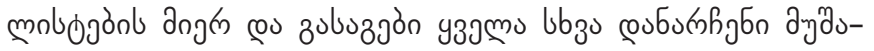
zolvonzol.

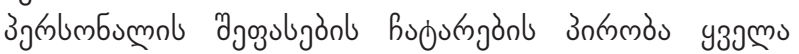

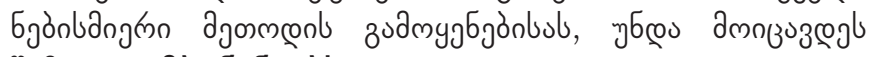

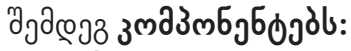

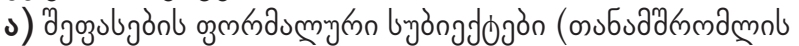

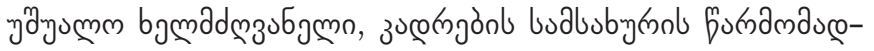
उјб

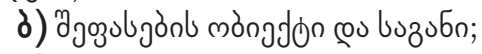

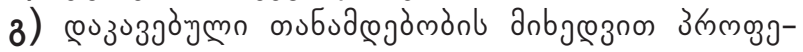

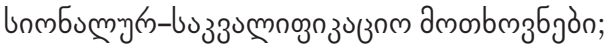

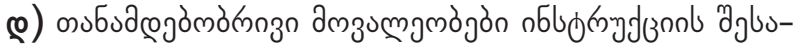

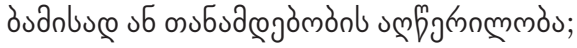

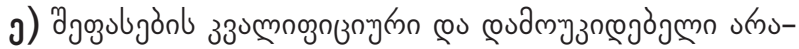

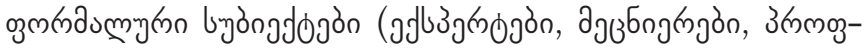

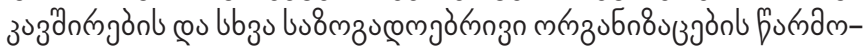
даœ

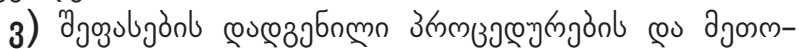

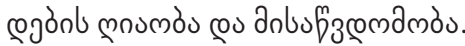

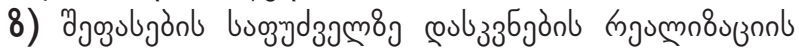
agปง

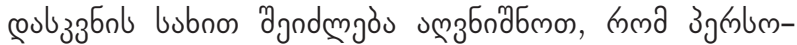

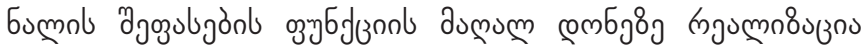

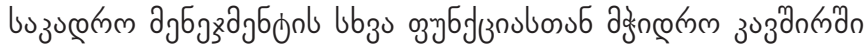

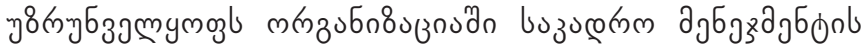

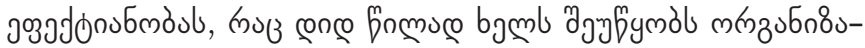

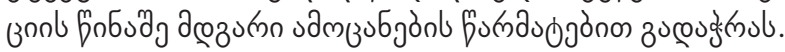

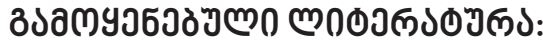

1. Коргова М. (2007). Кадровый менеджмент. Ростов-на-Дону.

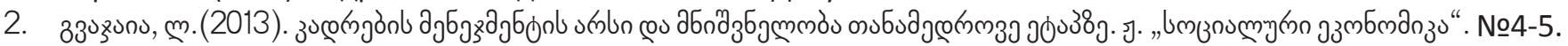




\section{ASSESSMENT OF THE WORKFORCE`S ACTIVITIES IS AN IMPORTANT FUNCTION OF STAFF MANAGEMENT}

LUARA GVAJAIA

https://doi.org/10.35945/gb.2017.04.021

Academic Doctor of Economics, Associated Professor of Georgian Technical University, Georgia

KEYWORDS: PERSONNEL, CONTROL, MANAGEMENT, ESTIMATION OF PERFORMANCE, TECHNIQUES, PRINCIPLES

\section{SUMMARY}

The article considers the basic problems of estimation of working performance of personnel, as part of the process of organization's personnel management. Functions of personnel management, factors that have impact on efficiency of estimation, the essence of estimation object, i.e. professional characteristic and skills are considered. The above-mentioned characteristics include the level of participation into solution of organizational problems and general organizational skills.

The basic principles of estimation of performance of personnel are determined, including objectivity, purposefulness, versatility, perfection, etc. The techniques of personnel estimation are considered, such as estimation with attestation, standard and self-estimation techniques. 